\title{
eJRIEPS
}

Ejournal de la recherche sur l'intervention en éducation physique et sport

$23 \mid 2011$

Varia

\section{Préambule eJRIEPS JAD 4}

\section{Serge Eloi et J-F Robin}

URL : http://journals.openedition.org/ejrieps/4532

DOI : 10.4000/ejrieps.4532

ISSN : 2105-0821

\section{Éditeur}

ELLIADD

\section{Référence électronique}

Serge Eloi et J-F Robin, « Préambule eJRIEPS JAD 4 », eJRIEPS [En ligne], 23 | 2011, mis en ligne le 01 juillet 2011, consulté le 21 février 2020. URL : http://journals.openedition.org/ejrieps/4532 ; DOI : 10.4000 /ejrieps. 4532

\section{(c) (1)}

La revue eJRIEPS est mise à disposition selon les termes de la Creative Commons Attribution 4.0 International License. 
La récente disparition de René Deleplace (9 janvier 2010), véritable pionnier de l'approche technologique, nous place face à une double responsabilité. La première a trait à la diffusion de sa pensée dans le respect des idées parfois très novatrices qu'il a su faire émerger ainsi que des concepts qu'il a dû formuler pour élaborer sa modélisation du rugby. La seconde fait référence à la nécessité de développer son travail et de le prolonger afin de pousser encore plus loin la compréhension des pratiques physiques humaines. Évidemment, nous l'avions invité aux quatrièmes journées Alain Durey (JAD 4). Un empêchement l'aura contraint à renoncer à ce séminaire. Nous nous faisions une joie de débattre en sa présence de l'extension de son travail de modélisation du rugby aux autres sports collectifs. Nous avons appris quelques jours plus tard qu'il faudrait désormais nous passer définitivement de ces remarques et contributions.

Les journées Alain Durey sont toujours le lieu de débats opiniâtres. Dans ceux qui se sont déroulés lors des JAD 4, nous avons pu apprécier toute la pertinence du point de vue de Jacques Rouyer, qui devait nous quitter dans les semaines qui suivirent (18 février 2010).

Les journées Alain Durey sont depuis 10 ans organisées sur le site du département STAPS de I'Université Paris Est Créteil (UPEC). Elles se déclinent en deux sessions. L'une sur l'approche technologique des Pratiques Physique Sportives et Artistiques (PPSA) et l'autre sur l'actualité des recherches en intervention pour l'Éducation Physique et le Sport (EPS). Nous publions ici les articles se rapportant uniquement à la première session. En effet, nous avons jugé plus opportun d'avoir un numéro thématique se rapportant exclusivement à l'analyse technologique des sports collectifs. Les articles issues des communications de la deuxième session sont parus dans le précédent numéro de la revue eJRIEPS (Monniotte, Vors, Mangeant).

Dans ce numéro de la revue eJRIEPS, nous nous situons dans la lignée des travaux de Deleplace $(1979,1983)$ mais aussi de ceux de Bouthier et Reitchess (1984), Bouthier et Durey (1994) ainsi que de nos propres travaux (Éloi \& Uhlrich, 2001).

Dans le premier article (Ulrich, Éloi \& Bouthier), les auteurs positionnent le concept de technologie dans le contexte de la recherche en STAPS. L'accent est particulièrement porté sur les registres de technicité.

Le deuxième article (Éloi \& Ulrich), définit la démarche technologique en mettant en relation les registres de technicité et les artefacts mobilisés aux différents niveaux de l'intervention. 


\section{eJRIEPS 23 avril 2011}

Le troisième article (Éloi) concrétise cette démarche technologique au travers de l'analyse du rôle de libéro en volley-ball.

Le quatrième article (Mouchet) consiste en une déclinaison du concept de registre de technicité dans le cadre de l'étude du rugby.

Le cinquième article (Amans-Passaga) a pour objectif la prise en compte de l'adversité dans le contexte du volley-ball scolaire.

Le sixième article (Lemoine) présente en football une analyse du jeu en déviation où la technique individuelle du joueur devient une maitrise collective « supérieure ».

Le septième article (Duprat) aborde l'influence de l'ouverture du score dans le résultat final en football lors de rencontres internationales.

Le dernier article (Bossard) s'appuie sur un travail de thèse récemment soutenue pour décrire l'activité décisionnelle de joueurs de football en situation de contre-attaque.

L'ensemble de ces articles a pour vocation la relance des réflexions à propos de l'analyse technologique dans le domaine des PPSA. C'est l'occasion de rappeler la place décisive que représente cet axe de recherche dans la compréhension des activités physiques humaines à l'heure de profondes mutations dans les formations en STAPS.

\section{Serge ÉLOI \& J-F ROBIN, éditeurs scientifiques invités}

